Accepted Manuscript

Fluid flow in wall-driven enclosures with corrugated bottom

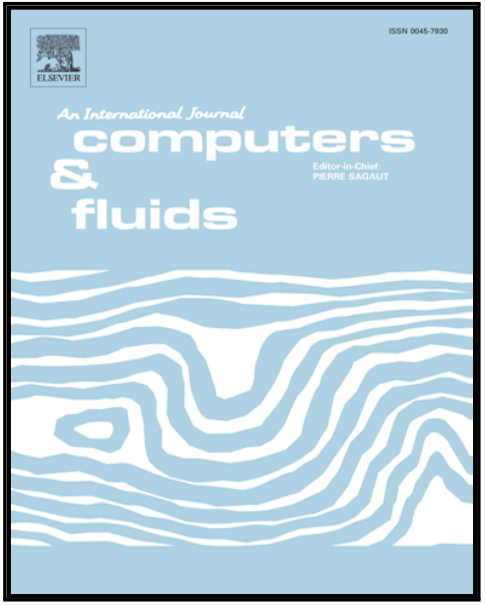

Manju Bisht, Sina Haeri, Dhiraj V. Patil

PII: S0045-7930(17)30126-3

DOI: 10.1016/j.compfluid.2017.04.008

Reference: CAF 3449

To appear in:

Computers and Fluids

Received date: $\quad 8$ October 2016

Revised date: $\quad 14$ March 2017

Accepted date: $\quad 7$ April 2017

Please cite this article as: Manju Bisht, Sina Haeri, Dhiraj V. Patil, Fluid flow in wall-driven enclosures with corrugated bottom, Computers and Fluids (2017), doi: 10.1016/j.compfluid.2017.04.008

This is a PDF file of an unedited manuscript that has been accepted for publication. As a service to our customers we are providing this early version of the manuscript. The manuscript will undergo copyediting, typesetting, and review of the resulting proof before it is published in its final form. Please note that during the production process errors may be discovered which could affect the content, and all legal disclaimers that apply to the journal pertain. 


\section{Highlights}

- LBM simulations for flow structures in two-dimensional wall-driven enclosures consisting of regular, square shaped, corrugations on the bottom wall.

- Counter-rotating eddies generate inside corrugations which depend upon Reynolds number.

- Flow transition Reynolds numbers found for left-most corrugation eddy merger event.

- Results are consistent with the literature for 2D flow inside slender and shallow cavity enclosures. 


\title{
Fluid flow in wall-driven enclosures with corrugated bottom
}

\author{
Manju Bisht, ${ }^{1}$ Sina Haeri, ${ }^{2}$ and Dhiraj V. Patil ${ }^{1, *}$ \\ ${ }^{1}$ School of Engineering, Indian Institute of Technology Mandi \\ Kamand, Mandi, Himachal Pradesh 175005, India \\ ${ }^{2}$ James Weir Fluids Laboratory, University of Strathclyde
}

Glasgow, Scotland, UK

(Dated: April 13, 2017)

\begin{abstract}
In this article, incompressible, continuum regime, viscous flow of Newtonian fluid in twodimensional (2D) wall-driven enclosures consisting of regular, square shaped, corrugations on the bottom wall is studied numerically. Steady and consistent simulation results are obtained using kinetic theory based lattice Boltzmann equation method (LBM) and solution of Navier-Stokes equation based on fictitious domain method (FDM). First, numerical validation is performed by comparing LBM and FDM results for velocity profiles at particular sections inside the enclosures and vertical velocity gradient at the top of the corrugation cavity. Flow features are then compared for variations in Reynolds number, bottom-wall corrugation height and number of these corrugations. Further, complex eddy dynamics with respect to input parameters and geometry is discussed in detail. Flow transition Reynolds numbers showing distinct flow behavior are found. The numerical results obtained are verified and appear to be consistent with the previously published results for $2 \mathrm{D}$ flow inside slender and shallow cavity enclosures.
\end{abstract}

PACS numbers: 02.70.Ns; 47.11.-j; 47.32.-y

\footnotetext{
*dhiraj@iitmandi.ac.in
} 


\section{INTRODUCTION}

The flow over corrugated surfaces is relevant in many scientific and engineering applications, such as mass and heat exchangers [1] and has been widely investigated in the past using numerical and experimental techniques. Benjamin [2] theoretically studied shearing flows bounded by a wavy-surface to determine the normal and tangential stresses on the boundary. Then, the phase relationships between these stresses and the wavy-surface elevation are found to explore several examples. Pan and Acrivos [3] analyzed the steady flows in rectangular deep cavities with the uniform translation of the top wall and the results may be extrapolated to understand flow inside deep corrugations. Savvides and Gerrard [4] used a finite-difference method to investigate the steady and unsteady axisymmetric laminar flows through a corrugated tube, for applications in medical diagnostics. They considered the effect of tube-wall corrugation size and Reynolds number on the steady flow pattern such as flow separation and evolution of separated region then computed the overall flow resistance. For unsteady flows, the effects of Reynolds number, oscillation frequency and the ratio of the mean volume flow rate to its amplitude were studied. For medical application work, an arterial waveform with six harmonics was considered to investigate the occurrence of stagnant regions in the corrugations and to find out whether there is a large resistance change in comparison with a plane cylindrical tube. Ralph [5] numerically solved oscillatory viscous flows in wayy-walled tubes and flow characteristics were analyzed for various Reynolds number and Strouhal number. Stone and Vanka [6] studied the developing flow and heat transfer in a wavy passage by a numerical solution of the two-dimensional, unsteady, flow and energy equations. They observed the effect of Reynolds number on the flow transition. When the flow is unsteady, the mixing of a core and the near wall-fluids is shown to increase, causing enhanced heat transfer rates. Niavarani and Priezjev [7] solved the Navier-Stokes (NS) equations to examine the incompressible viscous fluid flow over a periodically rough surface and analyzed the effective slip length and the wall shear stress corresponding to the corrugation height. Few experimental studies were also performed to understand flow over corrugated surfaces, e.g. Vlachogiannis and Bontozoglou [8], as well as, Luo and Pozrikidis [9] investigated laminar film flow along an inclined periodic surface with rectangular corrugations. Lastly, flows over inclined as well as corrugated surfaces have been studied in the context of multi-phase or multi-component physics $[10,11]$. 
In the past, three-dimensional (3D) flow simulations in a grooved channel have also been analyzed. Amon and Patera [12] performed simulations of the three-dimensional flow in a grooved channel. They found that the primary Tollmien-Schlichting wave instability is supercritical in contrast to plane Poiseuille flow. The two-dimensional wave becomes unstable to a three-dimensional excitation that is interpreted as a slightly detuned classical channel secondary instability. Nishimura and Kunitsugu [13] experimentally and numerically examined the three-dimensional steady flow in grooved channels (with different cavity lengths) at intermediate Reynolds numbers. The 3D effects are dominant near the side walls of the channel. A self-sustained oscillation due to Tollmien-Schlichting waves (as a primary instability) and a Taylor-Geortler-like vortical structure (as a secondary instability) produces the $3 \mathrm{D}$ instability at the bottom of the groove. In this article, discussion is restricted to flow inside a top wall-driven, two-dimensional (2D) enclosure consisting of rectangular shaped wavy corrugations at the stationary bottom wall. Two-dimensional computational domain is an approximation when the wall/boundary in the third dimension is placed at infinity. It is important to understand three-dimensional flows inside corrugated geometries however, these simulations constitute our future work.

The 2D geometrical configuration is simple however, it allows detailed analysis of flow features inside the corrugated carities of various heights with the flow Reynolds number. Here, two different numerical techniques: (a) Lattice Boltzmann Method (LBM) and (b) Fictitious Domain method (FDM) in order to validate the simulation results are used. LBM is a computationally convenient method utilizing the discrete form (in phase-space and time domain) of 'the fullBoltzmann equation' and has been applied to study the dynamics of fluid flows from continuum to slip flow regimes [14-18]. LBM can be easily implemented and large numbers of CPUs and GPUs may then be employed efficiently for high-performance computations [19-21]. Specially, the use of GPUs for computational fluid dynamics is popular in recent times [22]. Lin et al. [23] performed multiple-relaxation lattice Boltzmann simulations of transition in deep 2D lid-driven cavity using GPUs and Chang et al. [24] simulated the instability phenomenon within a three-dimensional cavity at various depth-width aspect ratios with multiple-relaxation LBM on GPUs. Fictitious Domain Method (FDM) [25-28] is generally used to find the solutions of partial differential equations (PDEs) for a complicated domain. Glowinski et al. [29] described FDM in detail for external incompressible viscous flow and readers are directed to other important references cited therein. 
In the past, LBM has been successfully applied to understand 2D or 3D fluid flows over wavy surfaces. For example, Brenner and group [30, 31] studied the effect of sinusoidal wavy surfaces for laminar lubrication using LBM to investigate the shear flows in lubrication as well as in prediction and understanding of tribological problems. Further, Varnik et al. [32] examined the influences of random wall roughness on flows in strongly confined (micro)-channels by using LBM. It is found that the decrease in channel width causes strong enhancement in the roughness-induced flow instability. The results also confirm the LBM reliability for complex unsteady flows. Patil et al. [33] analyzed wall-driven flows in 2D rectangular enclosures. Their results are useful in correlating the fluid flow patterns and corresponding variations with the bottom-wall corrugation height. Ding et al. [34] numerically simulated the incompressible viscous flow over several non-smooth surfaces using the lattice Boltzmann method. In the literature, to the best knowledge of the authors of this article, no prior work on the use of FDM to analyzefluid flow over wavy surface has been carried out.

The primary objective of this work to analyze the effects of corrugated bottom-wall surface on the flow structures for top-wall-driven 2D enclosures. First, a numerical validation is performed using LBM/FDM and then flow features are compared for variations in flow Reynolds number, corrugation height and number of these corrugations. In Section II, preliminary mathematical formulations for two methods employed is briefly described. In Section III, geometrical configurations, prescribed boundary conditions, problem set-up for the two numerical techniques, grid-independence studies, and comparison of LBM results with FDM for a representative case are detailed. In Section IV, numerical results for flow in two-dimensional wall-driven enclosures with rectangular bottom corrugations are presented. In Section V, a summary of this article is given.

\section{MATHEMATICAL FORMULATION}

In this section, two different numerical methods, namely, (A) Lattice Boltzmann Method: based on the kinetic theory principles for the solution of the discrete Boltzmann equation and (B) Fictitious Domain Method: for the solution of NS equations are briefly described. The following discussion on the mathematical formulation of these two methods is restricted considering their application for simulation of fluid flow in continuum regime only. 


\section{A. Lattice Boltzmann with BGK collision model}

The discrete lattice Boltzmann equation can be easily derived from the continuous Boltzmann equation [16]. LBM describes the time evolution of particle distribution functions, $f_{i}(\vec{x}, t)$ ( $i$ represents different discrete lattice directions) which define the probability that a fluid particle with velocity $\vec{e}_{i}$ enters the lattice site $\vec{x}$ at time $t$. The finite-difference approximation to the discrete lattice Boltzmann equation is given as,

$$
f_{i}\left(\vec{x}+\vec{e}_{i} d t, t+d t\right)-f_{i}(\vec{x}, t)=\Omega_{i}(\vec{x}, t), \mathrm{i}=0,1, \ldots, 8
$$

Here, $d t$ is the discrete time-interval and $\Omega_{i}$ is the collison operator. The effect of collisions could be accounted for using simplified models and one such model is the wellknown Bhatnagar-Gross-Krook (BGK) collision model [35], it is given as, $\Omega_{i}(\vec{x}, t)=$ $-1 / \tau\left[f_{i}(\vec{x}, t)-f_{i}^{e q}(\vec{x}, t)\right]$. Here, $f_{i}^{e q}(\vec{x}, t)$ is the Maxwellian equilibrium distribution function at $(\vec{x}, t)$ and $\tau$ is the single-relaxation time parameter which is related to the kinematic viscosity of the fluid. The relaxation time can also be understood as the average time interval between the particle collisions. It is known that upon application of the Chapman-Enskog expansion to the discrete LB equations, the NS equations are recovered. The kinematic viscosity $\nu$ of the fluid in terms of the relaxation time $\tau$ thus is estimated as, $\nu=(\tau-0.5) c_{s}^{2} / d t$. It is clear from this expression that in order to avoid negative viscosities the dimensionless relaxation time, $\tau$ must always be larger than $0.5[36]$. With the use of BGK collision approximation, for very large Reynolds numbers, the algorithm becomes numerically unstable, that is when $\tau$ is very close to 0.5 . Hence, other discrete versions of the collision operators are available in the literature $[37,38]$ which could then be applied instead of BGK approximation.

The Maxwellian-Boltzmann distribution function is used to define the discrete equilibrium distribution functions, $f_{i}^{e q}(\vec{x}, t)$ [18]. By using Taylor's series expansion (which is truncated up to the terms of second order in Mach number), the equilibrium distribution function is defined as follows,

$$
f_{i}^{e q}(\vec{x}, t)=w_{i} \rho\left[1+3 \frac{\vec{e}_{i} \cdot \vec{u}}{c^{2}}+\frac{9}{2} \frac{\left(\vec{e}_{i} \cdot \vec{u}\right)^{2}}{c^{4}}-\frac{3}{2} \frac{\vec{u} \cdot \vec{u}}{c^{2}}\right]
$$

where, $c=d x / d t=1$ being the unit lattice speed which is related to the lattice speed of sound as $c_{s}=c / \sqrt{3}$. For the 2D fluid flows, a standard D2Q9 square lattice model (that is 9 
number of particle velocities) is extensively been used with $w_{i}$ being the weights representing the masses related with the particles traveling on each lattice direction [16]. These weights are given as, $w_{0}=4 / 9, w_{1,2,3,4}=1 / 9$, and $w_{5,6,7,8}=1 / 36$.

The macroscopic quantities are computed at each discrete lattice location and discrete instantaneous time. These quantities are particle mass density, $\rho$ and the particle momentum density $\rho \vec{u}$ approximated using zeroth and first moment rules as,

$$
\rho(\vec{x}, t)=\sum_{i=0}^{8} f_{i}(\vec{x}, t), \quad \rho \vec{u}(\vec{x}, t)=\sum_{i=0}^{8} \vec{e}_{i} f_{i}(\vec{x}, t) .
$$

The fluid pressure, $p$, can then be estimated using computed density-field by employing the state equation in the lattice parameter set as $p=\rho c^{2} / 3$. Hence, the pressure is computed locally which implies that there is no need for solving the pressure-Poisson equation. The computations for the pressure-Poisson equation in the case of NS solution methods is timeconsuming and tedious. Further, due to the local operation of collisions (non-linear effects) and only the near-neighbour communications for the distribution function updates, LBM is a good candidate for the high-performance computing using large numbers of CPU and GPU cores.

\section{B. Fictitious Domain Method (FD)}

The FD hydrodynamics solver used in this study is explained and validated elsewhere [39]. The numerical framework was later extended for the simulations of conjugate heat transfer phenomena $[28,40]$ and is capable of exploiting the GPU technology to provide up to a 10fold speedup [41]. In this paper, however, an overview of the numerical method is provided for completeness. The corrugations can be generated by placing rigid objects with densities $\rho_{o}$ within a square cavity. From a computational point of view, these are presented by another set of Lagrangian (or material) grid points superimposed on a background Cartesian grid. A fluid with a density $\rho_{f}$ and a viscosity $\mu_{f}$ is assumed to fill the cavity. A single set of momentum and continuity equations on the whole domain $\Omega_{d}$-including the rigid objects - can be written as [28]

$$
\begin{gathered}
\frac{\partial \rho u_{i}}{\partial t}+\frac{\partial \rho u_{i} u_{j}}{\partial x_{j}}=-\frac{\partial P}{\partial x_{i}}+\frac{\partial}{\partial x_{j}}\left[\mu_{f}\left(\frac{\partial u_{i}}{\partial x_{j}}\right)\right]+f_{i} \\
\frac{\partial \rho}{\partial t}+\frac{\partial \rho u_{i}}{\partial x_{i}}=0
\end{gathered}
$$


subject to the following rigidity constraint within objects' domain $\Omega_{o}$ :

$$
\frac{1}{2}\left(\frac{\partial u_{i}^{R}}{\partial x_{j}}+\frac{\partial u_{j}^{R}}{\partial x_{i}}\right)=0, \forall x \in \Omega_{o} .
$$

The mixture density $\rho$ is defined by

$$
\rho=\Theta_{o} \rho_{o}+\left(1-\Theta_{o}\right) \rho_{f}
$$

where $\Theta_{o}$ is a step function equivalent to the volume fractions, which assumes a value of one in the object domain $\Omega_{o}$ and zero otherwise. The volume forcing term $f_{i}$ in Eq. 4 is an extra force on the object's domain $\Omega_{o}$ that enforces the rigidity constraint (Eq. 6) which can be written as

$$
f_{i}=\rho_{o} \frac{u_{i}^{R}-u_{i}^{l}}{\Delta t} .
$$

In Eq. 8, $u_{i}^{R}$ is the rigid body velocity and $u_{i}$ is a velocity at current iteration calculated from Eq. 4 on the Eulerian grid. In this paper, only stationary objects are considered and $u_{i}^{R}$ is simply zero; therefore

$$
f_{i}=-\rho_{o} \frac{u_{i}^{\ell}}{\Delta t}
$$

However, an interpolation scheme is required to transfer variables between the Eulerian background grid and the grid that is used to represent the objects. This is done using discrete delta functions and the reader is referred to [28] for further information.

\section{PROBLEM DEFINITION}

The simulation of fluid flows in 2D wall-driven enclosures with the corrugated bottom surface is performed using two numerical techniques, namely, LBM and FDM (explained in earlier Section II). Here, regularly spaced corrugations on the bottom-wall surface (a rectangular wave-form) have been considered. Flow patterns are then analyzed for the effect of various parameters such as flow Reynolds number, the height of the bottom-wall surface corrugations as well as the wavelength of these corrugations.

\section{A. Problem setup for LBM}

In the present work, a 2D square computational domain (with $L_{=1} \times H_{=1}$ ) consisting of $N \times N ;(N=256)$ uniform lattice (or grid) points is used. The bottom-wall is kept stationary having wavelength corrugations of two representative heights $(h=0.25$ and $h=0.75)$ as 


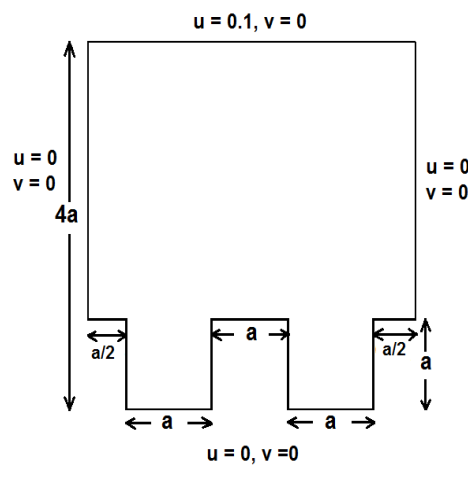

(a) Two wavelength

corrugation with $h=0.25$

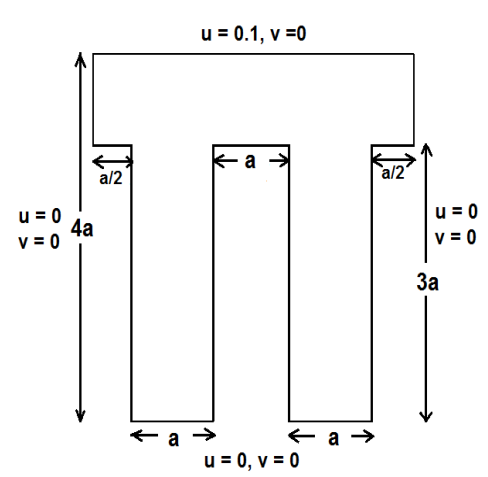

(b) Two wavelength corrugation with $h=0.75$

FIG. 1: Geometry and Boundary conditions for different wavelength corrugations. At the top and bottom walls of enclosure diffuse-reflection LB boundary conditions and at remaining solid walls simple bounce-back boundary conditions are applied.

shown in Fig. 1. The considered wavelengths for these corrugations are $\lambda=0.5$ and $\lambda=0.25$. The flat upper (or top) wall moves from left to right with a uniform lattice (non-dimensional) velocity of $U=0.1$. The Reynolds number for LBM simulations is defined as $\operatorname{Re}=U N / \nu$, where $N$ is the characteristic length of the domain consisting of total lattice nodes on top-wall and $\nu$ is non-dimensional lattice kinematic viscosity of the fluid. The flow-field is established by the shearing stress which is exerted on the adjacent fluid layer by the moving upper-wall surface. Initially, fluid velocities at all lattice nodes (except the top nodes on the upper-wall surface) are set to zero with a uniform non-dimensional lattice fluid density being $\rho=1.0$. Simulations are carried out for Re ranging from 100 to 3200. In order to simulate very high Re flows, finer grid resolution is necessary for numerical stability. If one employs the BGK approximation, a large number of lattice points are required (cell Reynolds number for BGK model is around 15) or advanced collision models are encouraged to be used [37, 38, 42]. The numerical results obtained using LBM simulations are compared with that from FDM for consistency and accuracy. Further, new and interesting flow features are reported and discussed in the following section. On the solid wall boundaries (i.e. left and right side wall surfaces), a no-slip boundary condition has been applied. For the LBM simulations, diffuse reflection boundary condition is used on top and bottom-walls and at the remaining solid-walls, non-equilibrium bounce-back has been applied [43, 44]. 


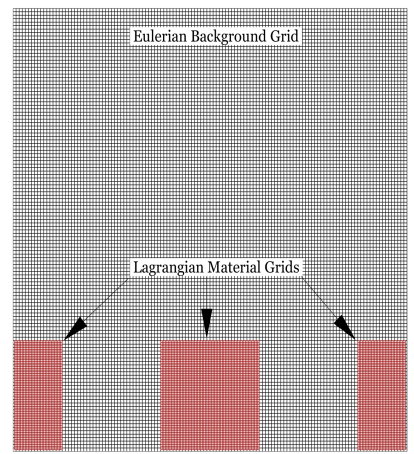

FIG. 2: A representation of the FD problem setup. The number of grid points is reduced for better presentation. The material and Eulerian grid points are identified with red and black lines, respectively.

\section{B. Problem setup for FDM}

The FDM problem set-up is similar to the LBM simulations geometrically. An $N \times$ $N ;(N=256)$ Cartesian background grid is used. Depending on the wavelength of corrugations, $\lambda$, the number of corrugations in the domain of $\left(L_{=1} \times H_{=1}\right)$ can be calculated as $N_{c}=2,4$. Consequently, another set of Lagrangian grids is overplayed on the background grid in order to specify $N_{c}+1$ objects within the domain, see Fig. 2. The size of the material grid is chosen to provide 9 points within each Eulerian control volume. The top-wall (lid) uniform velocity is kept to a normalized value of 2 .

\section{Grid independency test}

The grid independence test for LBM simulations is performed with the use of two lattice grid sizes, $256^{2}$ and $512^{2}$ and for the highest value of $\operatorname{Re}=3200, h=0.75$, with $\lambda=$ 0.5. Figure 3 shows the streamline plot comparison for the steady-state solutions obtained for both these grid sizes. It is clear from the streamline plots that the solution is not grid sensitive with an overall averaged error of $3.75 \%$ between mesh $256^{2} \& 512^{2}$. The characteristics of eddy motions were found consistent. Hence, it is expected that the gridindependency holds good for the lower range of Re. Therefore, a grid size of $256^{2}$ is selected for the LBM simulations reported here. Similarly, for FDM simulations, $256^{2}$ uniform mesh size is used. 


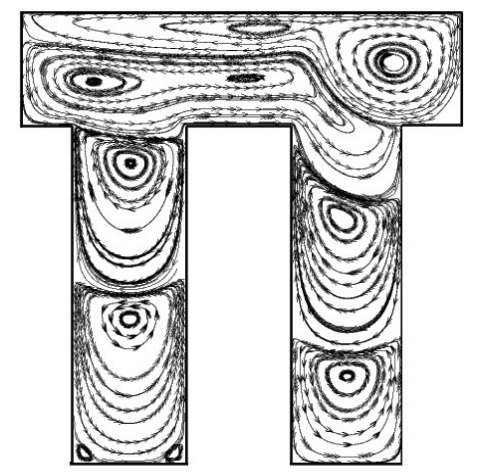

(a) Corrugation with $\lambda=0.5,256^{2}$ nodes

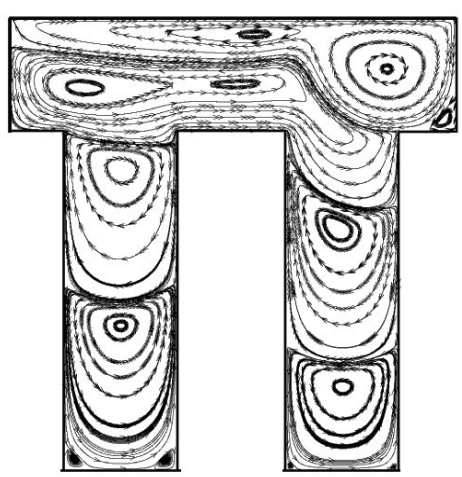

(b) Corrugation with $\lambda=0.5,512^{2}$ nodes

FIG. 3: Grid independence test: Streamline plots for $\mathrm{Re}=3200$ with an averaged error of $3.75 \%$ in between $256^{2}$ grid points and $512^{2}$ grid points.

\section{Comparison of LBM and FDM results}

The 2D wall-driven flow inside an enclosure and with corrugated bottom-wall has not been reported previously in the literature. Hence, in this work two different numerical techniques, namely LBM and FDM, are used for comparison of simulation results. The comparison of velocity profiles obtained at specific locations and for $\operatorname{Re}=100$ and $\operatorname{Re}=3200(\lambda=0.5$, $h=0.25)$ is depicted in Fig. 4. The normalized $u$-velocity profiles along $y$ at $x=\lambda / 2$ and $x=3 \lambda / 2$ are shown in Figs. 4a and $4 \mathrm{~b}$, respectively. Further, shown in Figs. 4c and $4 \mathrm{~d}$ are the normalized $v$-velocity profiles along $x$ at $y=h / 2$ and $y=(h+1) / 2$, respectively. Next, Fig. 5 illustrates the variation between LBM and FDM results for $u$-velocity gradient in $y$-direction which is computed at $h=0.25$ and $\operatorname{Re}=3200, \lambda=0.5$. It is seen that the LBM results are in excellent agreement with the FDM results.

\section{RESULTS AND DISCUSSIONS}

A number of simulations have been carried out in order to analyze fluid flow in the wall-driven $2 \mathrm{D}$ enclosure with a specified bottom-wall surface corrugations. Various characteristics of the flow in the corrugated cavity are examined by exploring the effects of variations of Reynolds number Re, the wavelength of corrugations, $\lambda$ and height of corrugations, $h$. The streamline plots are plotted and analyzed for eddy dynamics and structures. Re is varied from 100 to 3200 , a number of wavelength corrugation considered on the bottom surface are two and four, each with a height of $h=0.25$ and 0.75 , respectively. 


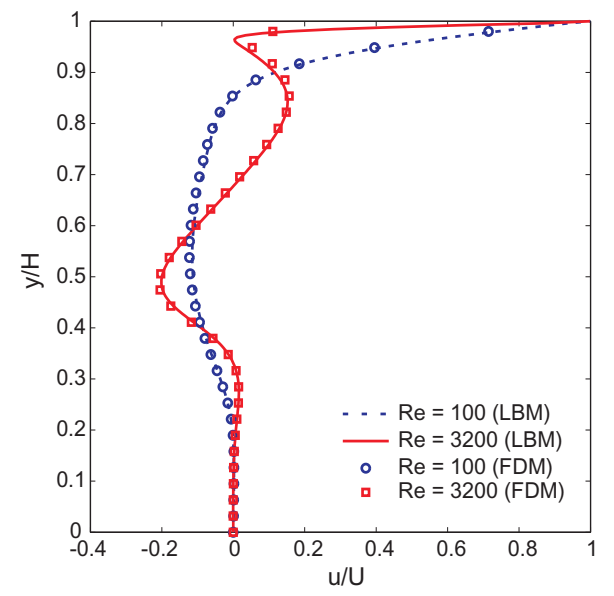

(a) $u$ velocity along $y$ at $x=\lambda / 2$

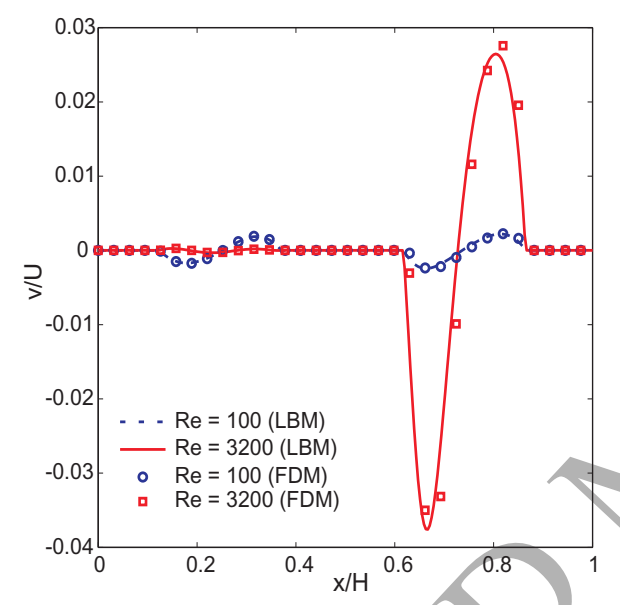

(c) $v$ velocity along $x$ at $y=h / 2$

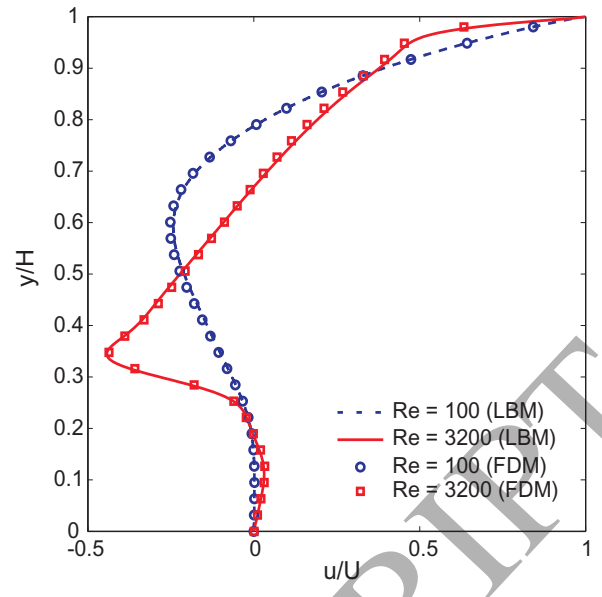

(b) $u$ velocity along $y$ at $x=3 \lambda / 2$

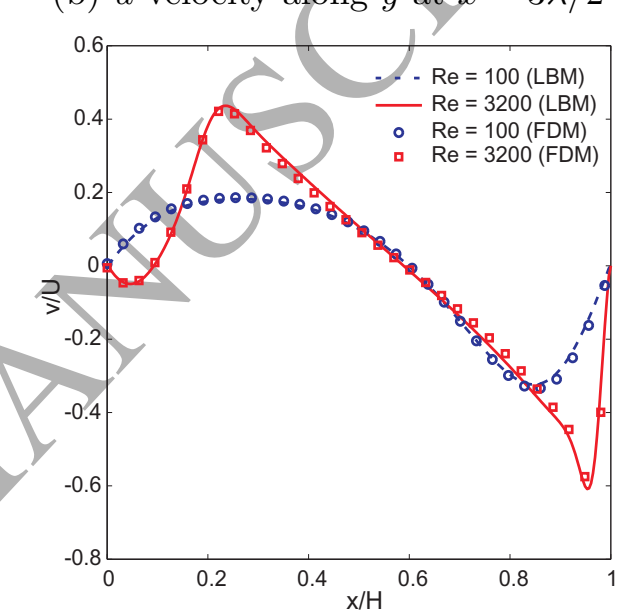

(d) $v$ velocity along $x$ at $y=(h+1) / 2$

FIG. 4: Comparison of results obtained from LBM and FDM simulations: Sectional velocities for 2 wave corrugations, $\mathrm{Re}=100$ and $\mathrm{Re}=3200$.

\section{A. Bottom wall-corrugations with $h=0.25$ and for $\lambda=0.5$ :}

First, the influence of Reynolds number is discussed and which is observed to be pronounced on the flow structure. Figure 6 shows streamline plot for two-wave corrugations with $h=0.25$, as a steady-state solution $\left(L_{2}\right.$ error norm $\left.<10^{-14}\right)$. For a moderate corrugation and $\mathrm{Re}=100$, a large primary eddy $(\mathrm{PE})$ is developed occupying the major portion of the cavity, which is similar to the plane bottom-wall square cavity flow [45]. In addition to the PE, the flow inside corrugated legs of bottom-wall (similar to the rectangular deep cavity flow) form a single secondary eddy in each corrugation leg (as $h=0.25$ ). The 


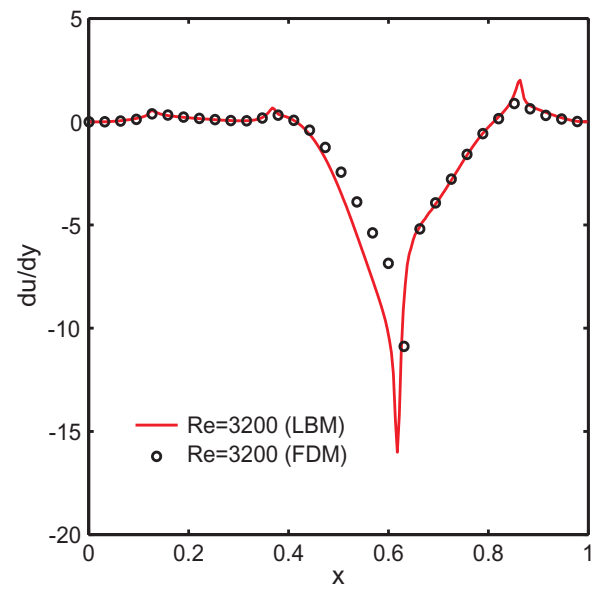

FIG. 5: Comparison between LBM and FDM results of $u$-velocity gradient in $y$-direction computed at $h=0.25, \lambda=0.5$ for $\operatorname{Re} \rightleftharpoons 3200$.

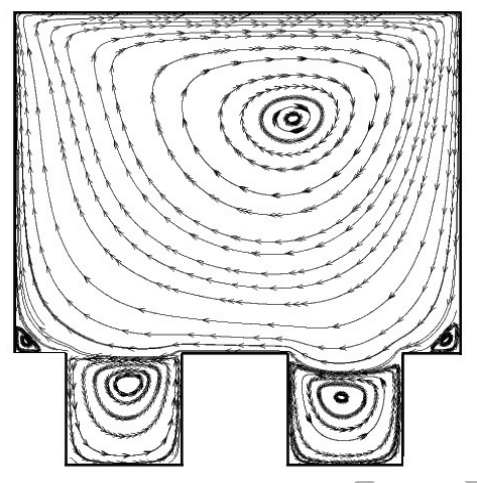

(a) $\operatorname{Re}=100$

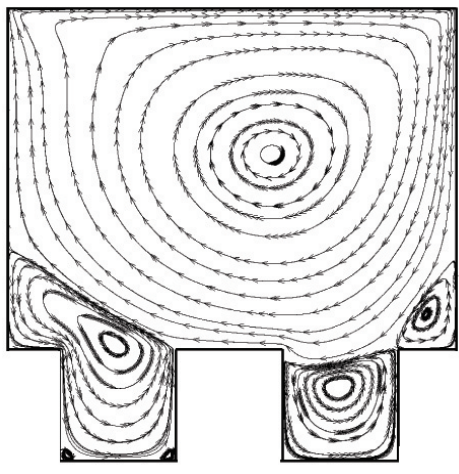

(b) $\operatorname{Re}=500$

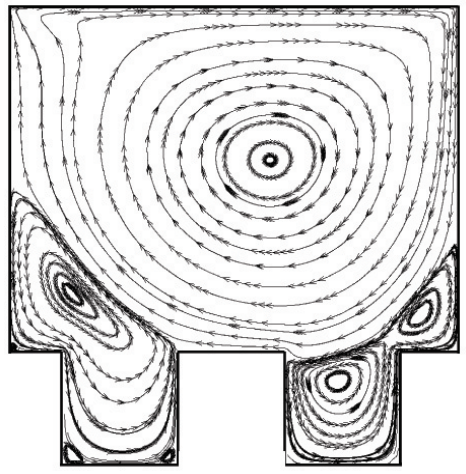

(c) $\operatorname{Re}=1000$

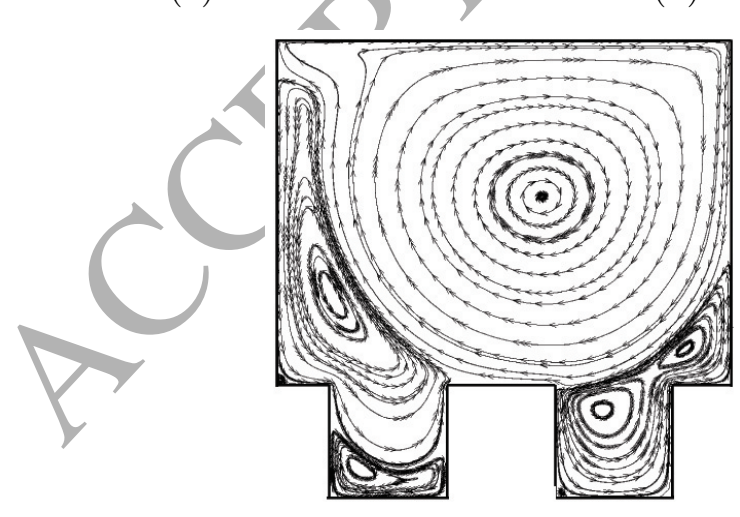

(d) $\operatorname{Re}=2000$

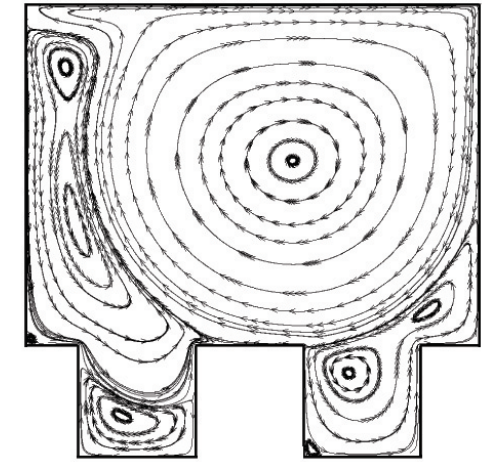

(e) $\mathrm{Re}=3200$

FIG. 6: Streamline plots for $\lambda=0.5, h=0.25$ corrugations with variation of Re. 
flow in these corrugations is driven by the shear of fluid on the top of the corrugation (i.e. above corrugation at height $h=0.25)$. Further, two eddies appear with the PE, one near the left corner and another located at the right corner of the cavity (Moffatt eddies [46]). Furthermore, at $h=0.25$ for next higher $\mathrm{Re}=500$, the secondary eddy inside the left-wall side corrugation leg merges with the left-wall corner eddy. Two minor eddies appear at the left and the right corner of the left-wall side corrugated bottom. This is in accordance with previously published results on eddy merger in deep cavities [33]. This phenomena ís due to an increased inertia of the primary eddy itself. It is observed that the right corner eddy which is attached to the right-wall grows in its size.

Next, for $\mathrm{Re}=1000$, the merged left-wall eddy grows further towards the moving wall. On the other hand, the right-wall corner eddy merges with the secondary eddy of the rightside corrugation leg. Further, for high values of Reynolds number ( $\operatorname{Re}=2000$ and 3200), the growth of the merged eddy located at the left-wall continues, forming distinct secondary eddies at the bottom of the left corrugation leg. However, the merged eddy situated at the right-wall remains approximately to be of same size due to an increased inertia of PE.

In order to obtain further understanding on the effect of Reynolds number and corresponding vortex structure, the vorticity contours for $h=0.25$ and $\lambda=0.5$ are plotted in Fig. 7. As Re increases, the primary eddy is observed to be like an inviscid vortex, that is, of the type of a solid-body rotation. This is due to formation of thin boundary layers along the solid walls with the central core in almost inviscid motion.

Figure 8 shows the kinetic energy contours for $\lambda=0.5$ with $h=0.25$ and variations in Re. The kinetic energy is maximum near the top wall (moving wall) of the enclosure and diminishes inside the corrugation legs and in the shallow region above corrugations. It is observed that the kinetic energy of the corner eddies increases with the Re. The order of kinetic energy magnitude close to the primary eddy centre is $1 \times 10^{-3}$ and inside the corrugations vary between $1 \times 10^{-5}$ and $1 \times 10^{-9}$. From the kinetic energy plot, range of flow velocity values as well as position of eddy centers could be estimated.

Figure 9 shows the steady-state streamline contours corresponding to variations of $\operatorname{Re}=$ 220, 250, 280, 300, 315 for $h=0.25$ and $\lambda=0.5$. These simulations are carried out to find flow transition Reynolds number which exhibit distinct flow regimes. Here, the analysis is restricted to eddy dynamics inside the leftmost corrugation leg. It is to be noted that the transition flow Reynolds number corresponding to other similar eddy dynamics (for 


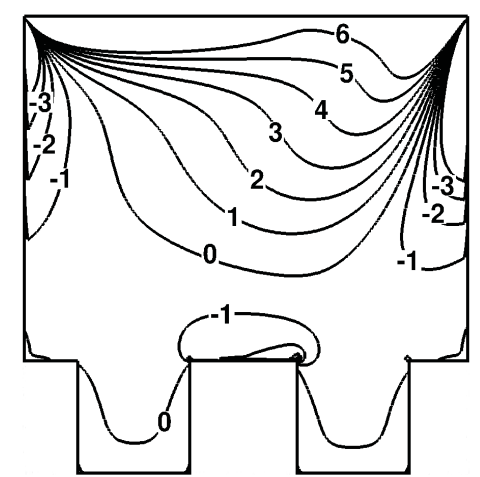

(a) $\operatorname{Re}=100$

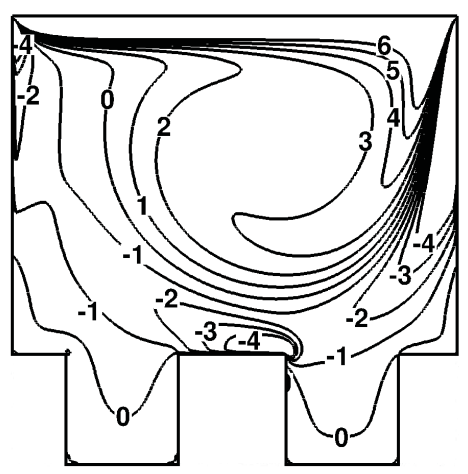

(b) $\operatorname{Re}=500$

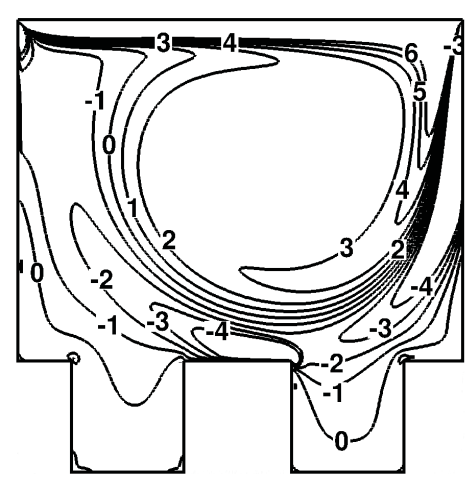

(c) $\operatorname{Re}=1000$

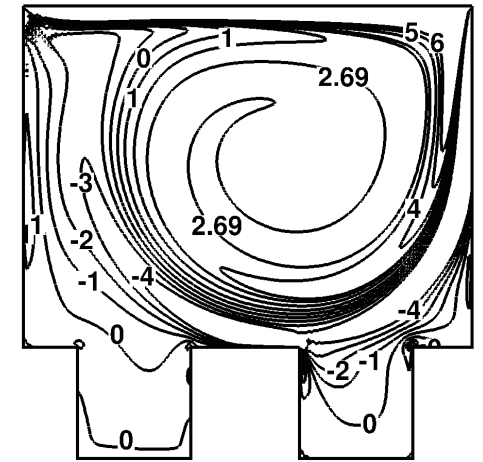

(d) $\operatorname{Re}=2000$

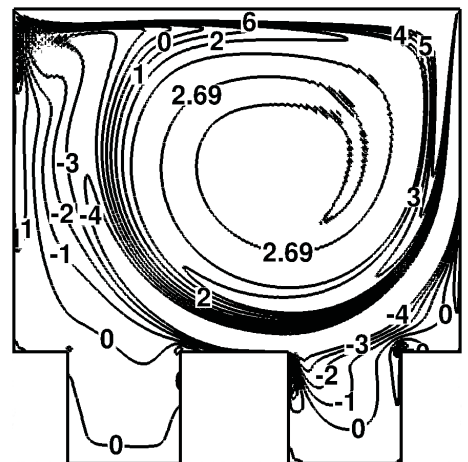

(e) $\operatorname{Re}=3200$

FIG. 7: Vorticity contours for $\lambda=0.5, h=0.25$ corrugations with variation of Re.

example, transition Re between flow structures for $\mathrm{Re}=1000$ and $\mathrm{Re}=2000$, refer Fig. 6) is different. In this article, the estimation of other flow transition Re is refrained. From Fig. 9, the merging of the secondary eddy inside the left corrugation leg and the corner eddy attached to the Teft wall of the enclosure is clearly evident. When $R e=250$, these two eddies start interacting with each other. With the further increase in Re, these eddies enlarge and form a single secondary eddy at $R e=315$. This is the transition Re for the merger of two eddies for the given parameter space.

B. Bottom wall-corrugations with $h=0.25$ and for $\lambda=0.25$ :

The streamline plots for corrugations corresponding to $\lambda=0.25$ with a height of $h=0.25$ are shown in Fig. 10. It is observed that the secondary eddy at the left corner starts growing with the Reynolds number which is very similar to the eddy dynamics observed for $\lambda=0.5$. 


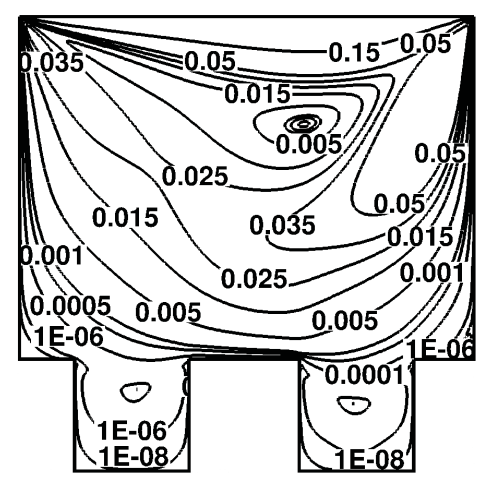

(a) $\operatorname{Re}=100$

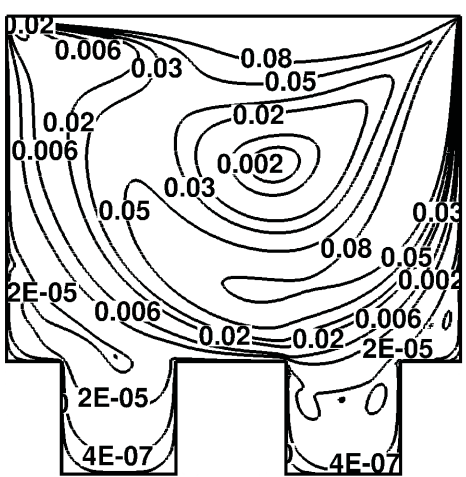

(b) $\operatorname{Re}=500$

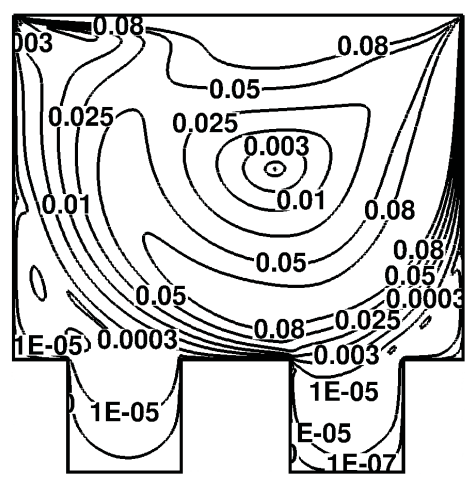

(c) $\operatorname{Re}=1000$

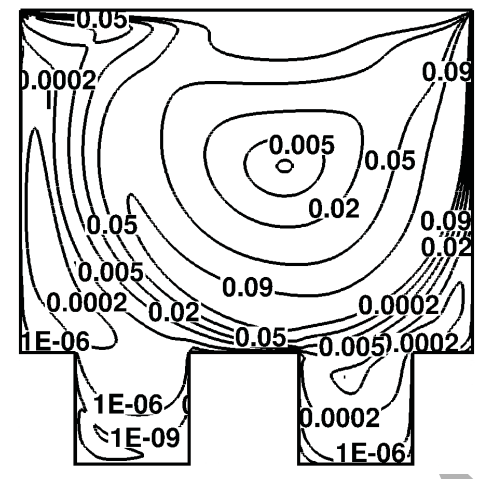

(d) $\operatorname{Re}=2000$

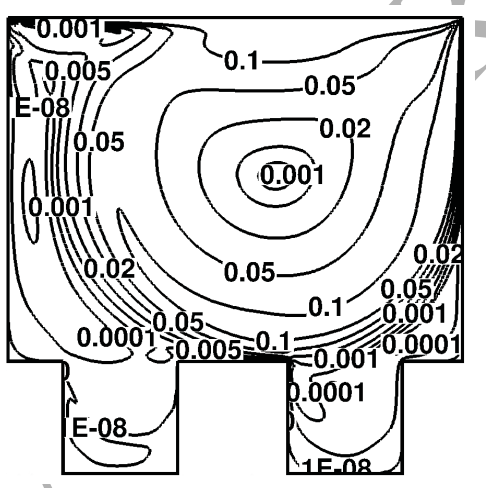

(e) $\operatorname{Re}=3200$

FIG. 8: Kinetic energy contours for $\lambda=0.5, h=0.25$ corrugations with variation in Re.

Further, from Fig. 10, it may once again be concluded that the Reynolds number has a pronounced effect on the fluid flow structure inside the enclosure. At $\operatorname{Re}=100$, the two secondary eddies are observed in each corrugation leg and two minor corner eddies appear above the corrugation height of $h=0.25$, one is located at the bottom left corner attached to the left boundary and another at the bottom right corner of the right boundary. At $\mathrm{Re}=500$, the secondary eddy emerges from the leftmost corrugation and merges with the secondary eddy present at the left corner. Further, the secondary eddy emerges from the rightmost corrugation and merges with the right corner eddy. It is observed that when Re increases from 500 to 1000, the left-wall attached merged eddy starts interacting with the secondary eddy of the second corrugation leg from the left-wall side. The combined eddies which are reattached to the left and the right walls grow upwards and hence two eddies (which are enlarged Moffatt corner eddies) appear at the bottom of the leftmost corrugation leg. With the increase in Re to 2000 and 3200, the left-wall attached eddy keeps grow in 


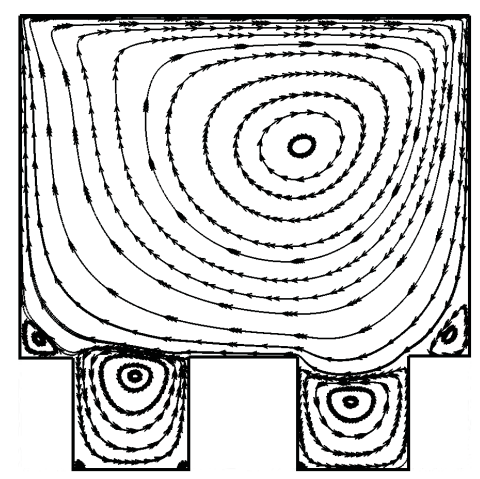

(a) $\operatorname{Re}=220$

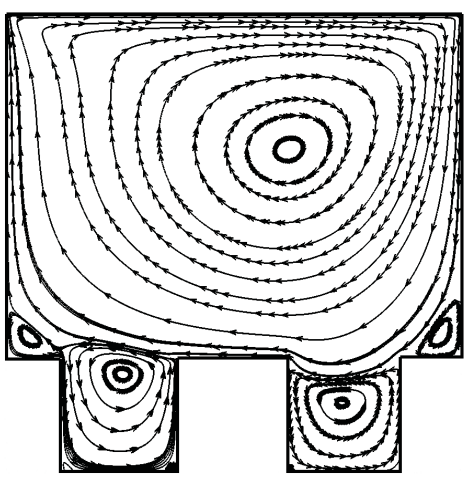

(b) $\operatorname{Re}=250$

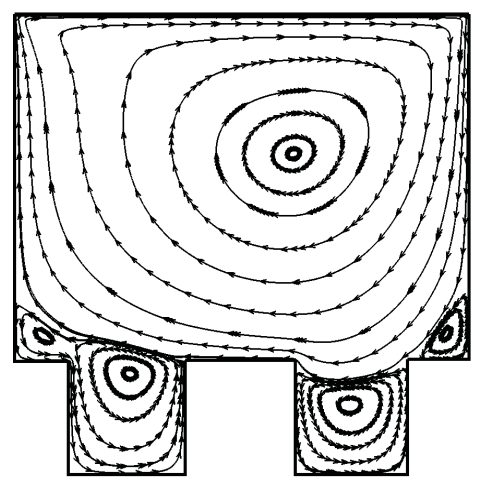

(c) $\operatorname{Re}=280$

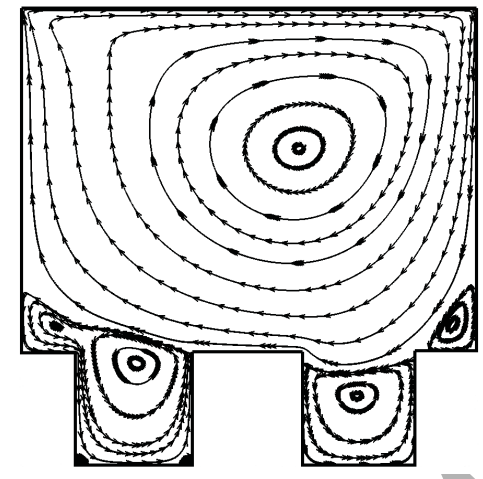

(d) $\operatorname{Re}=300$

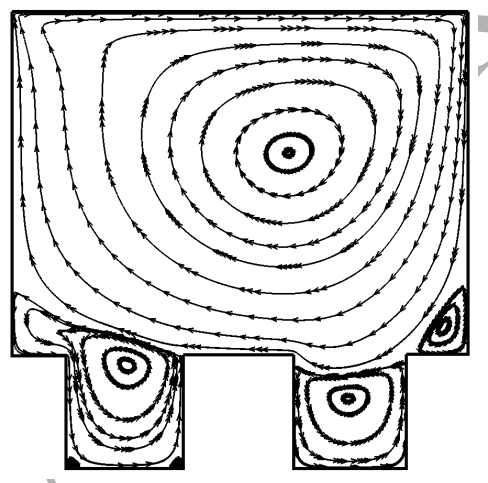

(e) $\operatorname{Re}=315$

FIG. 9: Streamline plots showing merger of attached to the left-wall and secondary eddy of the $\lambda=0.5, h \neq 0.25$ corrugations, finding flow-transition Re.

size towards moving wall. This phenomenon was explained in [33]. However, the right-wall attached eddy does not grow in size primarily due to the large fluid inertia at high Re. This observation is consistent to the eddy dynamics observed for the bottom wall-corrugations of $\lambda=0.5$ as per the discussion in the previous section. Now, it is clear that with the increase in Re, the left-wall attached eddy starts growing towards the upper-wall.

The effect of the number of bottom-wall corrugations for same height on the flow structure may also be analyzed by inspecting Figs. 6 and 10. For $\lambda=0.5$ corrugations, at $\operatorname{Re}=100$, only a single secondary eddy in each corrugated leg is seen whereas for $\lambda=0.25$ corrugations, two secondary eddies appear at each corrugation leg. Both $\lambda=0.25$ and $\lambda=0.5$ corrugations (for $\mathrm{Re}=500$ ) show that the secondary eddy from the leftmost corrugation leg moves out and merges with the corner eddy present at the left-side corner before starting of the corrugation leg at $h=0.25$. Further, in the case of $\lambda=0.25$ corrugations, the corner 


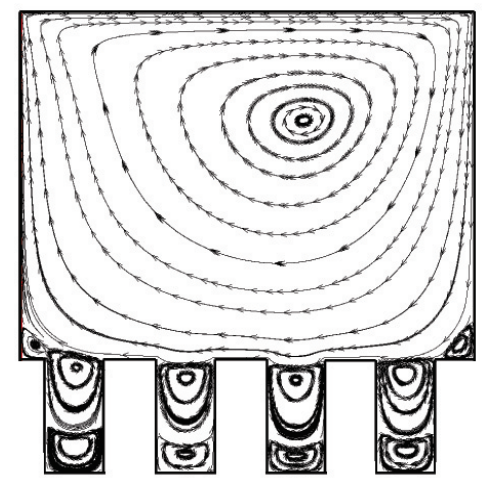

(a) $\operatorname{Re}=100$

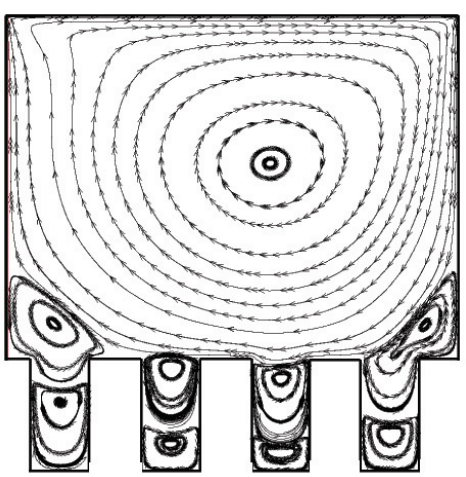

(b) $\operatorname{Re}=500$

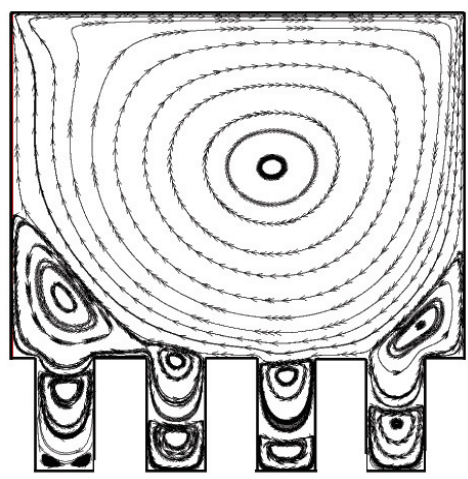

(c) $\operatorname{Re}=1000$

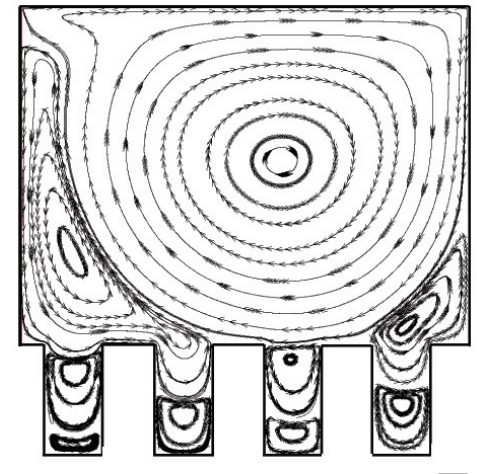

(d) $\mathrm{Re}=2000$

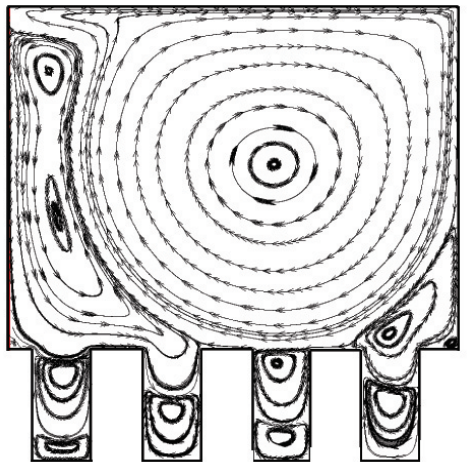

(e) $\operatorname{Re}=3200$

FIG. 10: Streamline plots for $\lambda=0.25, h=0.25$ corrugations with variation of Re.

eddy at the right-wall of enclosure combines with the secondary eddy present at the rightmost corrugation leg. This is distinctly different than $\lambda=0.5$ corrugations. At $\operatorname{Re}=1000$, the attached eddy present at the left-wall starts growing (as seen earlier) and merges with the secondary eddy of the second corrugation leg from the left-wall. Further, at $\mathrm{Re}=2000$ and 3200, the attached left-wall eddy grows quickly.

\section{Table I, Place Here.}

Hence, the distance of the left-wall attached eddy centre from the top boundary decreases with increasing Reynolds number. Table I shows the characteristics (centre location and vorticity at the centre) of the wall-attached eddy present at the left wall-boundary with respect to the flow Reynolds number for $\lambda=0.25$ and $\lambda=0.5$ corrugations with the height of $h=0.25$. Further, the characteristics of the first secondary eddy in both type of corrugations with $h=0.25$ are represented in the Tables II and III, respectively. 


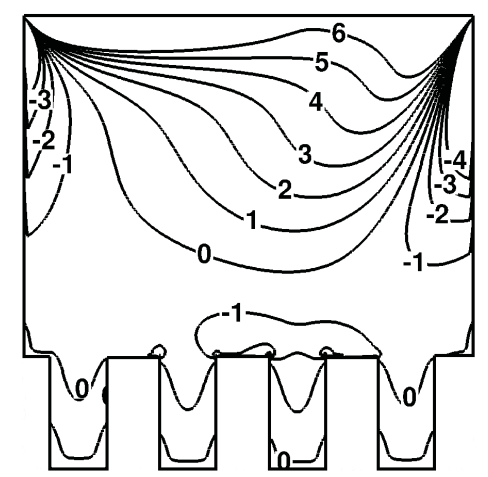

(a) $\operatorname{Re}=100$

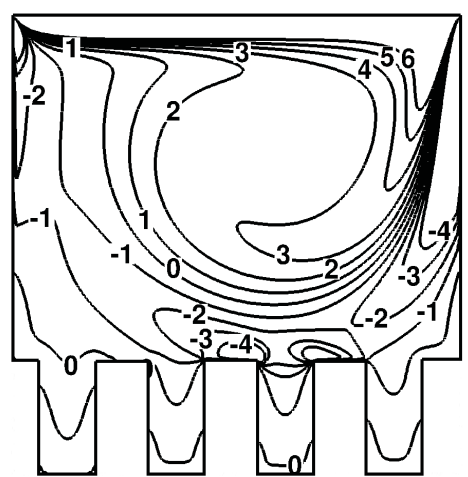

(b) $\operatorname{Re}=500$

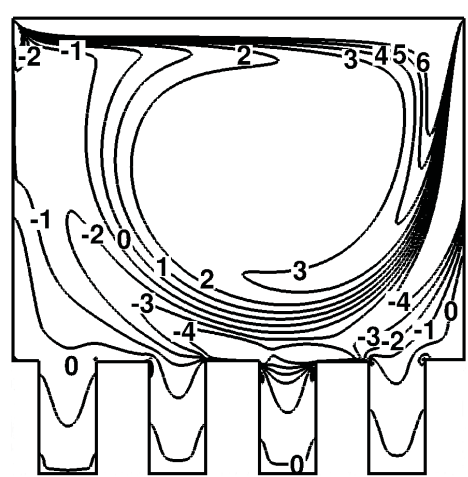

(c) $\operatorname{Re}=1000$

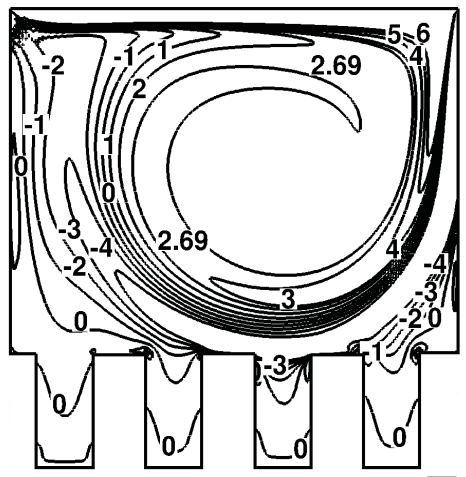

(d) $\operatorname{Re}=2000$

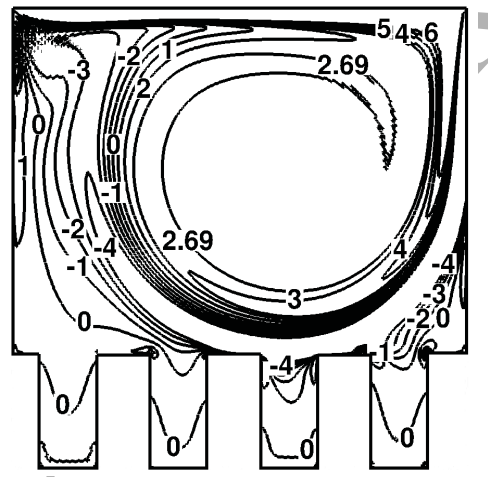

(e) $\operatorname{Re}=3200$

FIG. 11: Vorticity contours for $\lambda=0.25, h=0.25$, corrugations with variation in Re.

$$
\text { Table II, Place Here. }
$$

Table III, Place Here.

The vorticity contours corresponding to the streamline plots for $h=0.25$ and $\lambda=0.25$ are shown in Fig. 11. The vorticity contours provide insight into the flow field features corresponds to variation in Re. As Re increases, the core fluid move as a solid body with uniform velocity as seen in the Fig. 7.

Figure 12 shows the kinetic energy contours for $\lambda=0.25, h=0.25$ and with variation of Re. As Re increases, the kinetic energy of the corner eddies increases. The magnitude of the kinetic energy inside the corrugation legs varies from $1 \times 10^{-9}$ to $1 \times 10^{-13}$.

It is important to note that the flow inside corrugation legs is driven by the fluid shear present at the corrugation height. The flow pattern may also be analyzed by $u$-velocity gradient along the $y$-direction. The variation in the velocity gradient (may also be related 


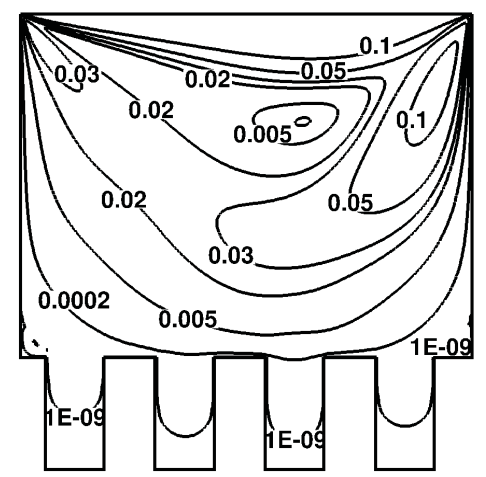

(a) $\operatorname{Re}=100$

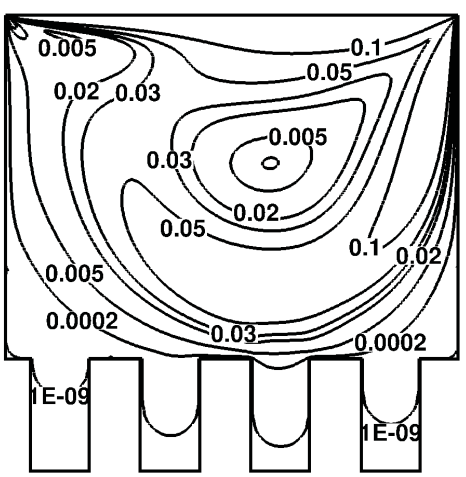

(b) $\operatorname{Re}=500$

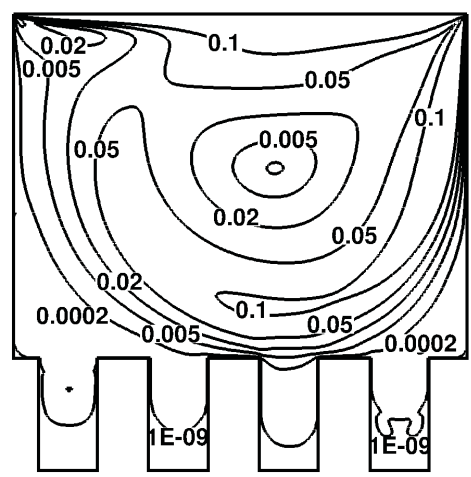

(c) $\operatorname{Re}=1000$

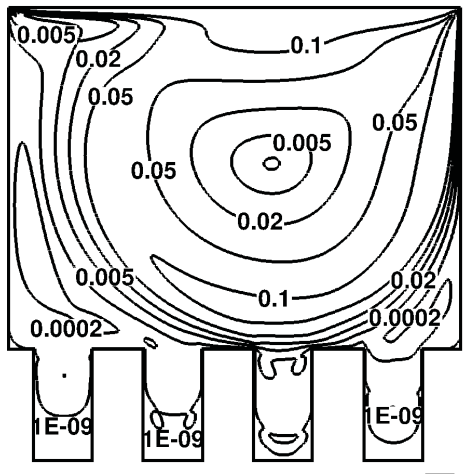

(d) $\operatorname{Re}=2000$

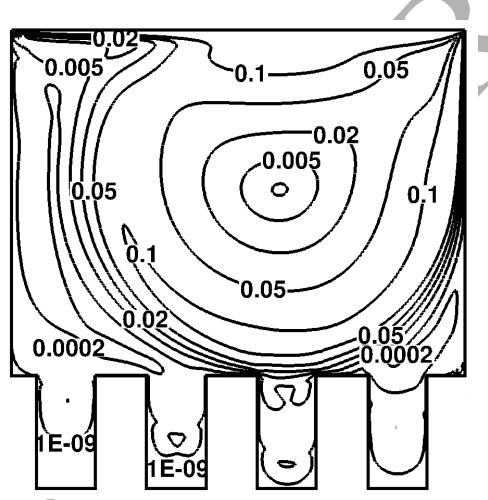

(e) $\operatorname{Re}=3200$

FIG. 12: Kinetic energy contours for $\lambda=0.25, h=0.25$ corrugations with variation in Re.

to shear stress component) at $h=0.25$ along the length of the enclosure for $\lambda=0.5$ corrugations with various Re are shown in Fig. 13. The scale on $y$-axis is logarithmic, and due to the clockwise movement of fluid, for the major portion of the length of enclosure, $\partial u / \partial y$ is negative, hence not seen in this plot. It is clear that with the increase in the flow Reynolds number, an eddy that appears in the left and the right corners above corrugations grow in it's size. In Fig. 13, the arrow direction depicts this evolution of the corner eddy.

\section{Bottom wall-corrugations with $h=0.75$ :}

For a strong corrugation of wavelength $\lambda=0.5$ with the height of corrugation $h=0.75$, the behavior of fluid flow examined by streamline plots as represented in Fig. 14, the primary recirculating eddy squeezed and moves closer to the right wall because of the strong reduction in the flow area in the top portion of the enclosure. Initially, when Re is low (100), each 


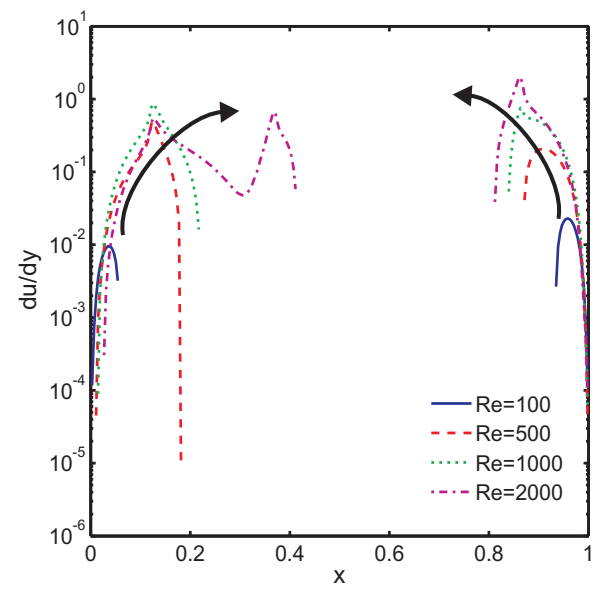

FIG. 13: Effect of Re on $u$-velocity gradient with respect to $y$ along the length of an enclosure for $\lambda=0.5$ and $h=0.25$ corrugations.

corrugation leg has two secondary eddies and a small eddy also appears at the left corner above corrugation of the enclosure. At $\operatorname{Re}=500$, the secondary eddies moves upwards due to moving top wall. Also, two minor secondary eddies formed in the left and the right corner of each rectangular corrugation bottom. When Re is more than 1000, the secondary eddies comes out from each wave and combines with the minor secondary eddy which appears at the left corner of the cavity.

The streamline plots for corrugation wavelength $\lambda=0.25$ and corrugation height $h=0.75$ with respect to various Reynolds number are shown in Fig. 15. When Re is 100, each corrugation leg have five secondary eddies. At Re $=1000$, the secondary eddy comes out from the third wave. When Reynolds number is more than 1000, the number of eddies above the corrugation starts increasing which brings more complex flow structures. It is observed that as the Re increases the reattached eddy at the left-wall moves towards the top boundary. For $R e=2000$ or 3200 , the bottom-most secondary eddy inside each corrugation shows some form of unsteadiness. However, the magnitudes of kinetic energy of these eddies are in the range of $1 \times 10^{-12}$ to $1 \times 10^{-13}$; showing a creeping flow regime. The fluid motion near lid and upper part of corrugation legs show steady behaviour. The unsteadiness may be attributed to the machine accuracy, grid-points as well as collision model employed. This aspect would warrant additional investigation.

The number of main secondary eddies in each rectangular corrugation are two for $\lambda=0.5$ corrugations and four for $\lambda=0.25$ corrugations at low values of $\operatorname{Re}(100)$. When $\operatorname{Re}$ is 500 , 
the corner eddies develops at the bottom of the corrugation for an enclosure with $\lambda=0.5$ corrugations, whereas in the case of $\lambda=0.25$ corrugations, the last eddy at the bottom of each corrugation grows upward. At $\operatorname{Re}=1000$, the secondary eddy from third corrugation leg coming out for $\lambda=0.25$ corrugations while for $\lambda=0.5$ corrugations, the corner eddies merge with each other at the bottom of corrugation leg. With $\operatorname{Re}=2000$ and 3200, in case of $\lambda=0.25$ corrugations, the secondary eddies coming from first three rectangular corrugation mixed with each other above the corrugation. On the other hand, in case of $\lambda=0.5$ corrugations, the secondary eddies coming out from both corrugation combined with each other.

Table IV, Place Here.

The characteristics of the reattached eddy at the left boundary for $\lambda=0.5$ and $\lambda=0.25$ corrugations with a height of $h=0.75$ is shown in Table IV. It is evident that as the Re increases the reattached eddy at the left-wall extends towards the top boundary. Also, the characteristics of the first secondary eddy in $\lambda=0.5$ and $\lambda=0.25$ corrugations with height $h=0.75$ are represented in the Table V and Table VI, respectively.

\section{SUMMARY}

Table V, Place Here.

Table VI, Place Here.

The flow of Newtonian fluid in two-dimensional wall-driven enclosures with the corrugated bottom-wall has been studied. The numerical simulations are done by the lattice Boltzmann method and verified with the results from Fictitious Domain method. The flow structures were discussed in detail. The computational results are investigated for various Reynolds numbers, corrugation heights and corrugation wavelengths and their effects on the flow pattern inside the corrugations as well as just above the corrugations were examined. The characteristics of eddy attached with the left-wall above the leftmost corrugation were also presented. It can be observed that the fluid move faster in the wall-driven enclosures as Re increases. The corrugation on the bottom wall of the enclosure reduces the area of the 


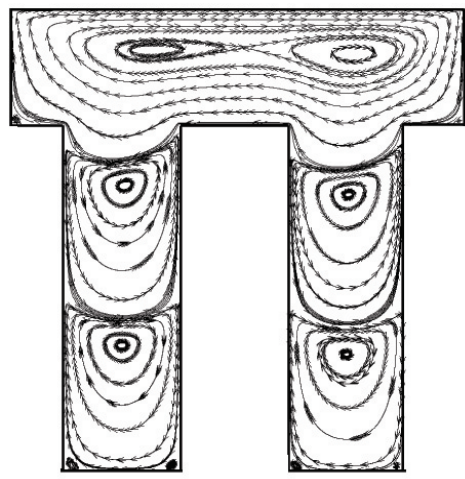

(a) $\operatorname{Re}=100$

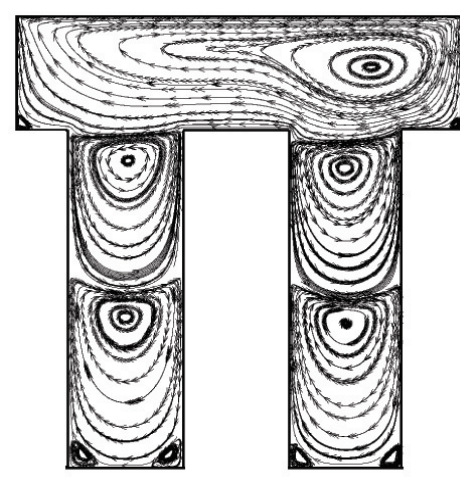

(b) $\mathrm{Re}=500$

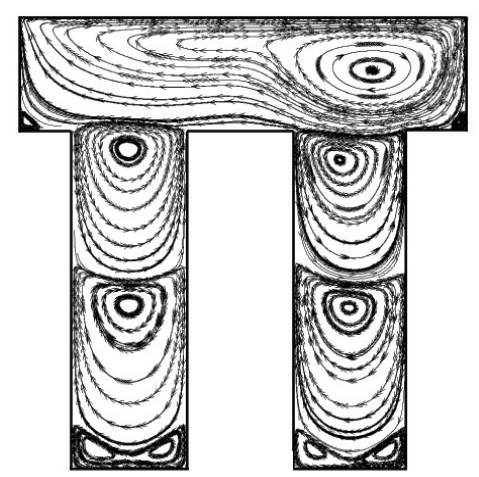

(c) $\mathrm{Re}=1000$

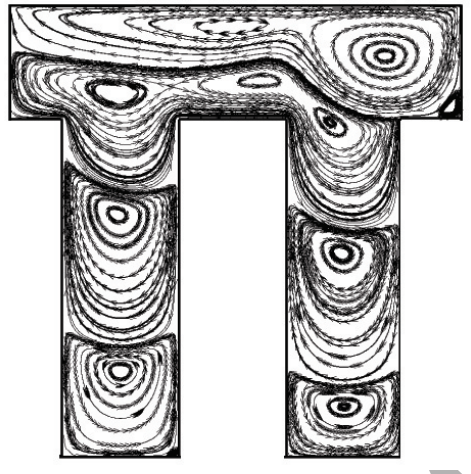

(d) $\operatorname{Re}=2000$

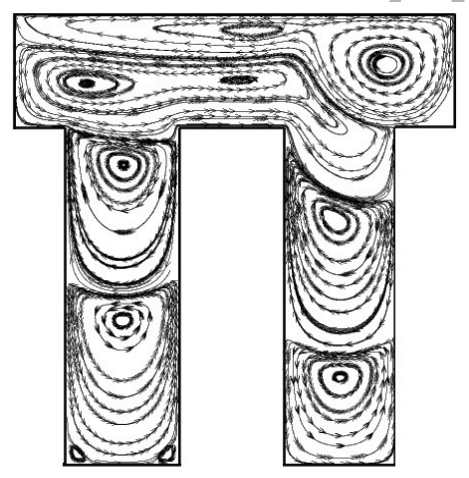

(e) $\operatorname{Re}=3200$

FIG. 14: Streamline plots for $\lambda=0.5, h=0.75$ corrugations with variation in Re.

cavity which causes to moye the centre of the primary eddy towards the right boundary and the top driving wall of the enclosure.

The following findings are derived from the present numerical results:

- For a fixed corrugation height, the increasing values of Re cause the reattached eddy at the left-wall to move towards the top wall. So, enhances its size and strength.

- The streamline plots showed that as Re increases, the inertia of the eddy attached with the right wall above the corrugations increases.

- For a fixed Re, the corrugation wavelength and corrugation height have a great effect on flow pattern and the number of eddies appeared inside the corrugations.

- At $\mathrm{h}=0.25$ and $\mathrm{Re}=100$, there are two secondary eddies inside the corrugation when $\lambda$ is 0.25 whereas there is a single secondary eddy inside the corrugation when $\lambda$ is 0.5 . 


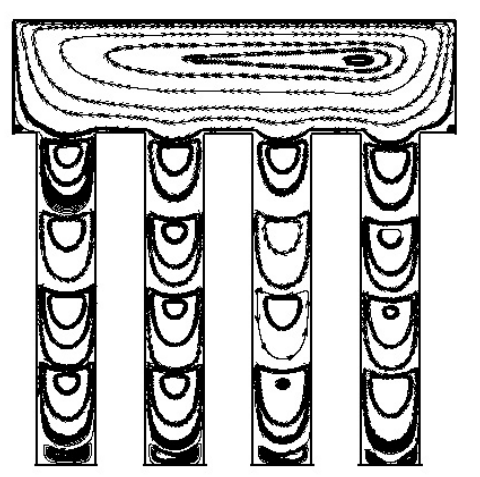

(a) $\operatorname{Re}=100$

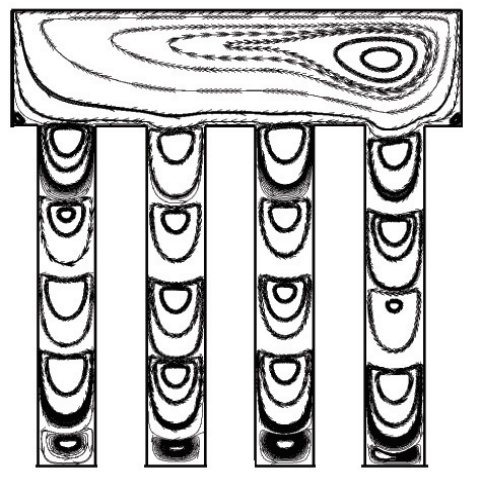

(b) $\operatorname{Re}=500$

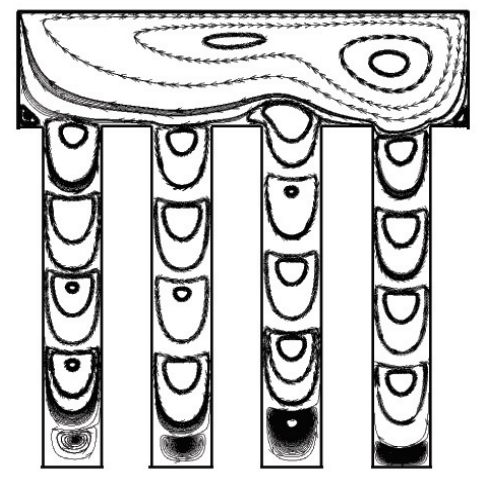

(c) $\mathrm{Re}=1000$

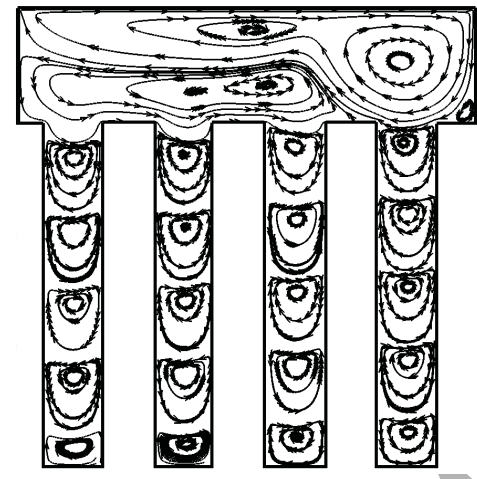

(d) $\operatorname{Re}=2000$

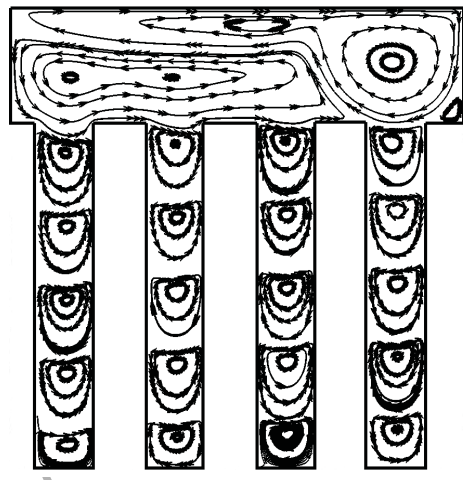

(e) $\operatorname{Re}=3200$

FIG. 15: Streamline plots for $\lambda=0.25, h=0.75$ corrugation with variation in Re.

- At $h=0.75$ and $R e=100$, there are four main secondary eddies inside the corrugation when $\lambda$ is 0.25 whereas there are two secondary eddies inside the corrugation when $\lambda$ is 0.5 .

- When $\lambda=0.5$ and $h=0.25$, two distinct flow transition Reynolds number were found between $\mathrm{Re}=100$ and $\mathrm{Re}=500$. At $\mathrm{Re}=250$, the secondary eddy inside the leftmost corrugation leg and the corner eddy above this corrugation leg starts interacting with each other. At $\operatorname{Re}=315$, these eddies merged together and forms a single eddy.

[1] Stasiek J. A. Experimental studies of heat transfer and fluid flow across corrugated-undulated heat exchanger surfaces. International Journal of Heat and Mass Transfer, 41:899-914, 1998. 
[2] Benjamin T.B. Shearing flow over a wavy boundary. Journal of Fluid Mechanics, 6:161-205, 1959.

[3] Pan F. and Acrivos A. Steady flows in rectangular cavities. Journal of Fluid Mechanics, 28: 643-655, 1967.

[4] Savvides C.N. and Gerrand J.H. Numerical analysis of the flow through a corrugated tube with application to arterial prostheses. Journal of Fluid Mechanics, 138:129-160, 1984.

[5] Ralph M.E. Oscillatory flows in wavy-walled tubes. Journal of Fluid Mechanics, 168:515-540, 1986.

[6] Stone K. and Vanka S.P. Numerical study of developing flow and heat transfer in a wavy passage. Journal of Fluids Engineering, 121:713-719, 1999.

[7] Niavarani A. and Priezjev N.V. The effective slip length and vortex formation in laminar flow over a rough surface. Physics of Fluids, 21:052105-1-052105-10, 2009.

[8] Vlachogiannis M. and Bontozoglou V. Experiments on laminar film flow along a periodic wall. Journal of Fluid Mechanics, 457:133-156, 2002.

[9] Luo H. and Pozrikidis C. Shear-driven and chamel flow of a liquid film over a corrugated or indented wall. Journal of Fluid Mechanics, 556:167-188, 2006.

[10] Kusumaatmaja H. and Vrancken R.J. and Bastiaansen C.W.M. and Yeomans J.M. Anisotropic drop morphologies on corrugated surfaces. Langmuir, 24(14):7299-7308, 2008.

[11] Sahu K.C. and Vanka S.P. A multiphase lattice Boltzmann study of buoyancy-induced mixing in a tilted channel. Computers \& Fluids, 50(1):199-215, 2011.

[12] Amon C.H. and Patera A.T. Numerical calculation of stable three-dimensional tertiary states in grooved-channel flow. Physics of Fluids A: Fluid Dynamics, 1(12):2005-2009, 1989.

[13] Nishimura T. and Kunitsugu K. Three-dimensionality of grooved channel flows at intermediate Reynolds numbers. Experiments in fluids, 31(1):34-44, 2001.

[14] Frisch U., Hasslacher B., and Pomeau Y. Lattice-gas automata for the Navier-Stokes equation. Physical Review Letters, 56:1505-1508, 1986.

[15] Qian Y.H., Succi S., and Orszag S.A. Recent advances in lattice Boltzmann computing. Annual Review of Computational Physics, 3:195-242, 1995.

[16] He X. and Luo L.S. Theory of the lattice Boltzmann method: from the Boltzmann equation to the lattice Boltzmann equation. Physical Review E, 56:R6811-R6817, 1997.

[17] He X. and Luo L.S. Lattice Boltzmann model for the incompressible Navier-Stokes equation. 
Journal of Statistical Physics, 88:927-944, 1997.

[18] Chen S.Y. and Doolen G.D. Lattice Boltzmann method for fluid flows. Annual Review of Fluid Mechanics, 30:329-364, 1998.

[19] Guo Z. and Shu C. Lattice Boltzmann Method and Its Applications in Engineering (Advances in Computational Fluid Dynamics). 3, 2013.

[20] Kaufman A. and Fan Z. and Petkov K. Implementing the lattice Boltzmann model on commodity graphics hardware. Journal of Statistical Mechanics: Theory and Experiment, 2009 (06):P06016, 2009.

[21] Hong P.-Y. and Huang L.-M. and Lin L.-S. and Lin C.-A. Scalable multi-relaxation-time lattice Boltzmann simulations on multi-GPU cluster. Computers \& Flujids, 110:1-8, 2015.

[22] Vanka S.P. and Shinn A.F. and Sahu K.C. Computational fluid dynamics using graphics processing units: challenges and opportunities. In ASME 2011 International Mechanical Engineering Congress and Exposition, pages 429-437. American Society of Mechanical Engineers, 2011.

[23] Lin L.-S. and Chang H.-W. and Lin C.-A. Multi relaxation time lattice Boltzmann simulations of transition in deep 2D lid driven cavity using GPU. Computers \& Fluids, 80:381-387, 2013.

[24] Chang H.-W. and Hong P.-Y. and Lin L.-S. and Lin C.-A. Simulations of flow instability in three dimensional deep cavities with multi relaxation time lattice Boltzmann method on graphic processing units. Computers \&f Fluids, 88:866-871, 2013.

[25] Glowinski R., Pan T.-W., and Periaux J. A fictitious domain method for Dirichlet problem and applications. Computing Methods in Applied Mechanical Engineering, 111:283-303, 1994.

[26] Khadra K., Angot P., Parneix S., and Caltagirone J.-P. Fictitious domain approach for numerical modelling of Navier-Stokes equations. International Journal for Numerical Methods in Fluids, 34:651-684, 2000.

[27] Glowinski R., Pan T.-W., Hesla T.I., Joseph D.D., and Periaux J. A fictitious domain approach to the Direct Numerical Simulation of incompressible viscous flow past moving rigid bodies: Application to particulate flow. Journal of Computational Physics, 169:363-426, 2001.

[28] Haeri S. and Shrimpton J.S. A new implicit fictitious domain method for the simulation of flow in complex geometries with heat transfer. Journal of Computational Physics, 237:21-45, 2013.

[29] Glowinski R., Pan T.-W., and Periaux J. A fictitious domain method for external incom- 
pressible viscous flow modeled by Navier-Stokes equations. Computing Methods in Applied Mechanical Engineering, 114:133-148, 1994.

[30] Brenner G., Al-Zoubi A., Mukinovic M., Schwarze H., and Swoboda S. Numerical simulation of surface roughness effects in laminar lubrication using the lattice Boltzmann method. Journal of Tribology, 127:603-610, 2007.

[31] Al-Zoubi A. and Brenner G. Simulating fluid flow over sinusoidal surfaces using the lattice Boltzmann method. Computers \& Mathematics with Applications, 55:1365-1376, 2008.

[32] Varnik F., Dorner D., and Raabe D. Roughness-induced flow instability: a lattice Boltzmann study. Journal of Fluid Mechanics, 573:191-209, 2007.

[33] Patil D.V., Lakshmisha K.N., and Rogg B. Lattice Boltzmann simulation of lid-driven flow in deep cavities. Computers \& Fluids, 35(10):1116-1125, 2006.

[34] Ding L., Shi W., and Luo H. Numerical simulation of viscous flow over non-smooth surfaces. Computers $\&$ Mathematics with Applications, 61:3703-3710, 2011.

[35] Bhatnagar P.L., Gross E.P., and Krook M. A model for collision processes in gases.I. Small amplitude processes in charged and neutral one-component systems. Physical Review, 94: $511-525,1954$.

[36] Qian Y.H., D'Humires D., and Lallemand P. Lattice BGK models for Navier-Stokes equation. Europhysics Letters, 17:479-484, 1992.

[37] Ning Y., Premnath K.N., and Patil D.V. Numerical study of the properties of the central moment lattice Boltzmann method. International Journal for Numerical Methods in Fluids, 82:59-90, 2016.

[38] Geier M., Schönhern M., Pasquali A., and Krafczyk M. The cumulant lattice Boltzmann equation in three dimensions: Theory and validation. Computers $\&$ Mathematics with Applications, $70(4): 507-547,2015$.

[39] S. Haeri and J. S. Shrimpton. On the application of immersed boundary, fictitious domain and body-conformal mesh methods to many particle multiphase flows. International Journal of Multiphase Flow, 40:38-55, 2012.

[40] S. Haeri and J. S. Shrimpton. A correlation for the calculation of the local Nusselt number around circular cylinders in the range $10 \leq \operatorname{Re} \leq 250$ and $0.1 \leq \operatorname{Pr} \leq 40$. International Journal of Heat and Mass Transfer, 59:219-229, 2013.

[41] S. Haeri and J. S. Shrimpton. Fully resolved simulation of particle deposition and heat transfer 
in a differentially heated cavity. International Journal of Heat and Fluid Flow, 50:1-14, 2014.

[42] Thantanapally C., Singh S., Patil D.V., S. Succi, and S. Ansumali. Quasiequilibrium lattice Boltzmann models with tunable Prandtl number for incompressible hydrodynamics. International Journal of Modern Physics C, 24(12):1340004, 2013.

[43] Succi S. The lattice Boltzmann equation for fluid dynamics and beyond. Oxford University Press, Oxford, 2001.

[44] Ansumali S., Karlin I.V., Frouzakis C.E., and Boulouchos K.B. Entropic lattice Boltzmann method for microflows. Physica A, 359:289-305, 2006.

[45] Patil D.V. and Lakshmisha K.N. Finite volume TVD formulation of lattice Boltzmann simulation on unstructured mesh. Journal of Computational Physics, 228(14):5262-5279, 2009.

[46] Moffatt H.K. Viscous and resistive eddies near a sharp corner. Journal of Fluid Mechanics, 18(01):1-18, 1964. 
TABLE I: Characteristics of the attached eddy to the left-wall for $\lambda=0.25$ and $\lambda=0.5$ corrugations with $h=0.25$. $(x, y)$ : centre of the attached eddy, $\omega$ : vorticity at the centre of the attached eddy

\begin{tabular}{ccccc}
\hline Re & $\lambda=0.5$ & \multicolumn{2}{c}{$\lambda=0.25$} \\
\hline & $(\mathrm{x}, \mathrm{y})$ & $\omega$ & $(\mathrm{x}, \mathrm{y})$ & $\omega$ \\
100 & $(0.0255,0.2777)$ & 0.0226 & $(0.0274,0.2807)$ & 0.0192 \\
500 & $(0.2348,0.2613)$ & 0.5445 & $(0.1052,0.3285)$ & 0.3159 \\
1000 & $(0.1397,0.3780)$ & 1.0296 & $(0.1116,0.3801)$ & 0.7623 \\
2000 & $(0.1182,0.4471)$ & 1.3362 & $(0.1160,0.4557)$ & 1.3995 \\
3200 & $(0.1052,0.5227)$ & 1.5313 & $(0.1052,0.5444)$ & 1.6157 \\
\hline
\end{tabular}

TABLE II: Characteristics of the first secondary eddy (SE) in each corrugation leg for $\lambda=0.5$ with $h=0.25$. $(x, y)$ : centre of SE, $\omega$ : vorticity at the centre of SE

\begin{tabular}{|c|c|c|c|c|}
\hline Re & & & \multicolumn{2}{|l|}{ 2nd SE } \\
\hline & & $\omega$ & $(\mathrm{x}, \mathrm{y})$ & $\omega$ \\
\hline & $(0.2521,0.1856)$ & 0.14597 & $(0.7297,0.1532)$ & 0.1447 \\
\hline & - & - & $(0.7341,0.1683)$ & 0.3890 \\
\hline 1000 & - & - & $(0.7276,0.1899)$ & 0.9063 \\
\hline 2000 & $(0.1808,0.0689)$ & -0.0086 & $(0.7125,0.1964)$ & 1.5882 \\
\hline 3200 & $(0.2089,0.0949)$ & -0.0172 & $(0.7103,0.1899)$ & 1.7231 \\
\hline
\end{tabular}




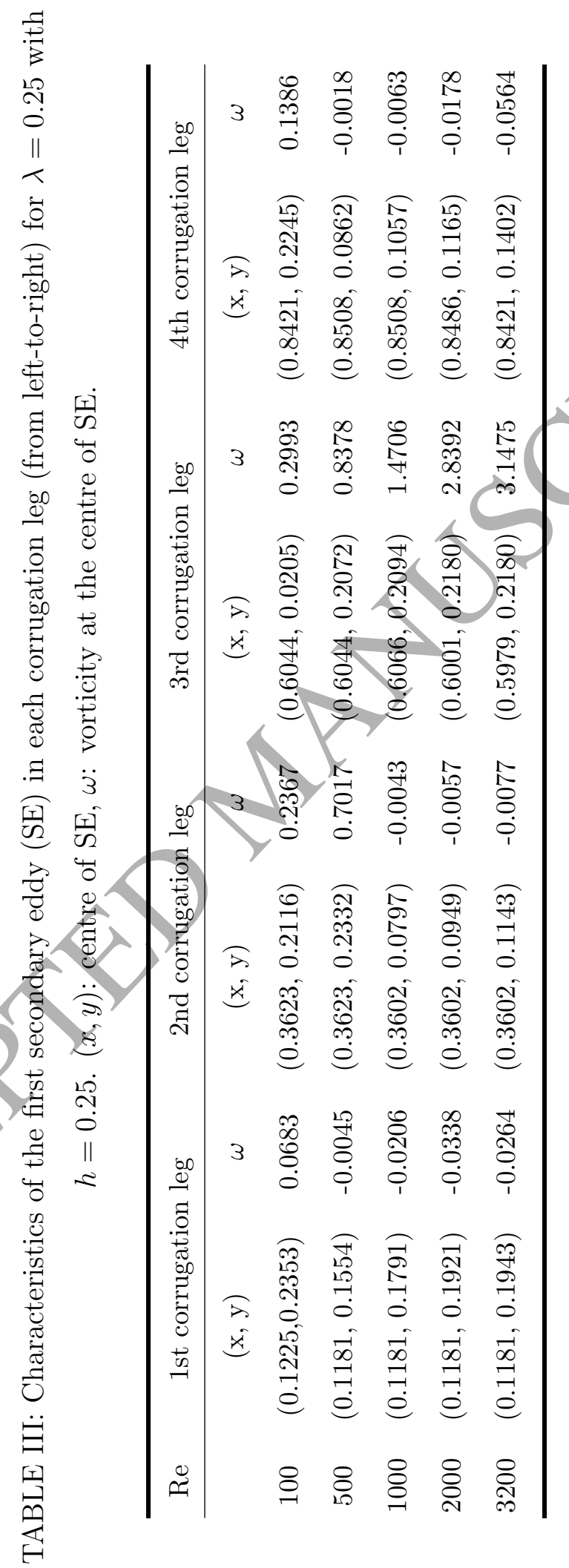


TABLE IV: Characteristics of the reattached eddy for $\lambda=0.5$ and $\lambda=0.25$ corrugations with height $h=0.75$. $(x, y)$ : centre of the reattached eddy, $\omega$ : vorticity at the centre of the reattached eddy

\begin{tabular}{ccccc}
\hline Re & $\lambda=0.5$ & \multicolumn{3}{c}{$\lambda=0.25$} \\
\hline & $(\mathrm{x}, \mathrm{y})$ & $\omega$ & $(\mathrm{x}, \mathrm{y})$ & $\omega$ \\
100 & $(0.0101,0.7626)$ & 0.0662 & $(0.0101,0.7605)$ & 0.0850 \\
500 & $(0.0101,0.7691)$ & 0.0299 & $(0.0122,0.7648)$ & 0.0309 \\
1000 & $(0.0144,0.7734)$ & 0.0289 & $(0.0166,0.7756)$ & 0.0346 \\
2000 & $(0.2305,0.8102)$ & 1.3970 & $(0.5416,0.8339)$ & 2.8961 \\
3200 & $(0.1614,0.8469)$ & 2.3554 & $(0.1289,0.8533)$ & 2.5517 \\
\hline & & & & \\
& & & &
\end{tabular}

TABLE V: Characteristics of the first secondary eddy in each corrugation for $\lambda=0.5$ with $h=0.75$. SE: secondary Eddy, $(x, y):$ centre of the reattached eddy, $\omega$ : vorticity at the centre of the reattached eddy

\begin{tabular}{|c|c|c|c|c|}
\hline & \multicolumn{2}{|l|}{$1 \mathrm{stSE}$} & \multicolumn{2}{|l|}{ 2ndSE } \\
\hline & $(\mathrm{x}, \mathrm{y})$ & $\omega$ & $(\mathrm{x}, \mathrm{y})$ & $\omega$ \\
\hline 100 & $(0.2499,0.6222)$ & 0.3153 & $(0.7427,0.6006)$ & 0.2823 \\
\hline 500 & $(0.2499,0.6848)$ & 0.5699 & $(0.7341,0.6675)$ & 1.9610 \\
\hline 1000 & $(0.2413,0.7129)$ & 0.4529 & $(0.7124,0.6870)$ & 3.2956 \\
\hline 2000 & $(0.2370,0.5400)$ & -0.0191 & $(0.7319,0.4558)$ & -0.0192 \\
\hline 3200 & $(0.2456,0.6697)$ & -0.3821 & $(0.7276,0.5487)$ & -0.0507 \\
\hline
\end{tabular}




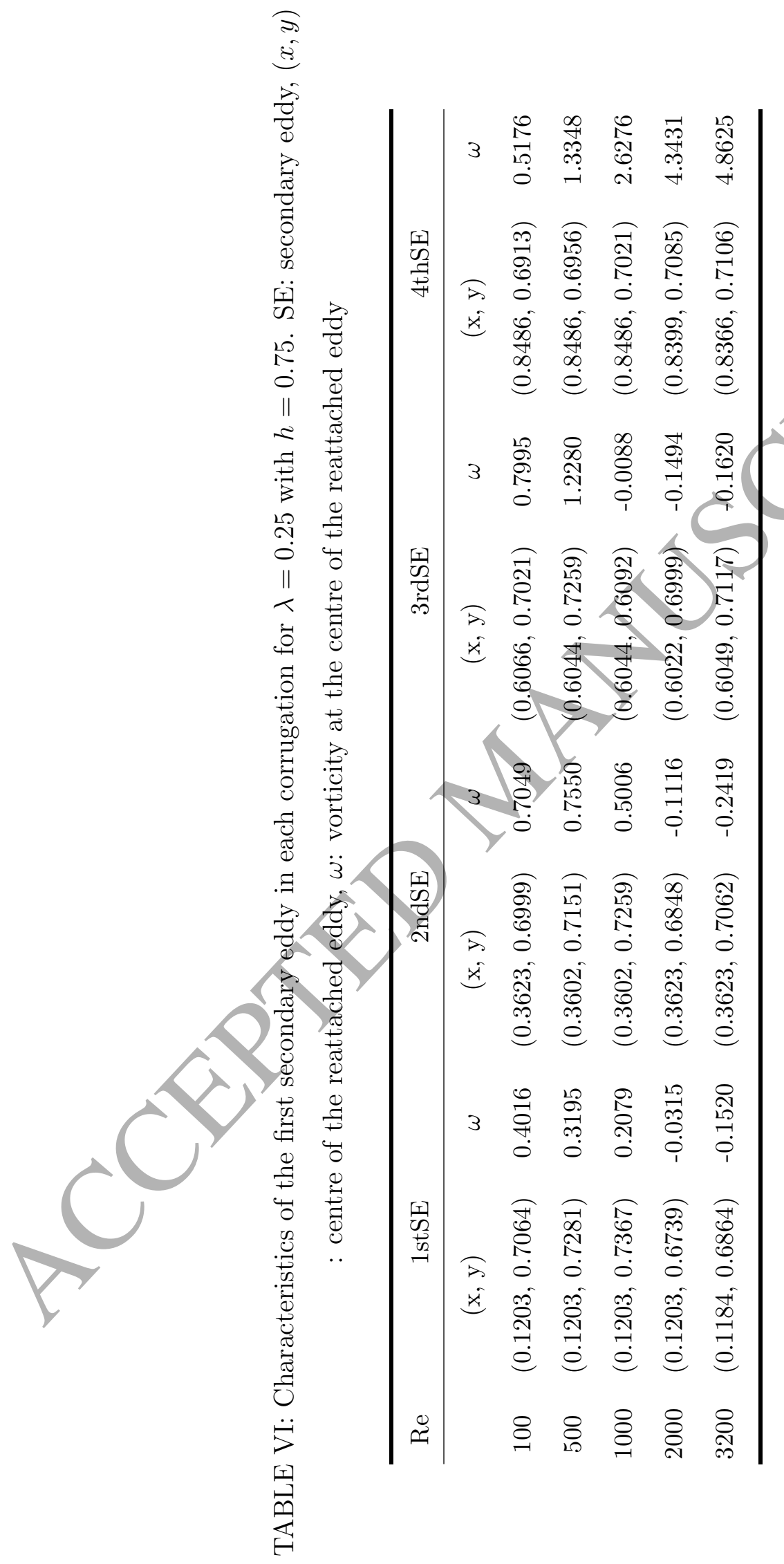

